\title{
Preparation of superconducting thin films of infinite-layer nickelate $\mathrm{Nd}_{0.8} \mathrm{Sr}_{0.2} \mathrm{NiO}_{2}$
}

\author{
Qiang $\mathrm{Gao}^{1}$, Yuchen Zhao ${ }^{1,2}$, Xingjiang Zhou ${ }^{1,2,3,4, *}$, and Zhihai Zhu ${ }^{1,2, *}$ \\ ${ }^{1}$ National Lab for Superconductivity, \\ Beijing National laboratory for Condensed Matter Physics, \\ Institute of Physics, Chinese Academy of Sciences, Beijing 100190, China \\ ${ }^{2}$ School of Physical Sciences, University of Chinese \\ Academy of Sciences, Beijing 100049, China \\ ${ }^{3}$ Beijing Academy of Quantum Information Sciences, Beijing 100193, China \\ ${ }^{4}$ Songshan Lake Materials Laboratory, Dongguan 523808, China \\ *Corresponding author: XJZhou@iphy.ac.cn, zzh@iphy.ac.cn
}

(Dated: March 17, 2021) 
The recent observation of superconductivity in thin films of infinite-layer nickelate $\mathrm{Nd}_{0.8} \mathrm{Sr}_{0.2} \mathrm{NiO}_{2}$ has received considerable attention. Despite the many efforts to understand the superconductivity in infinite-layer nickelates, a consensus on the underlying mechanism for the superconductivity has yet to be reached, partly owing to the challenges with the material synthesis. Here, we report the successful growth of superconducting infinite-layer $\mathrm{Nd}_{0.8} \mathrm{Sr}_{0.2} \mathrm{NiO}_{2}$ films by pulsed laser deposition and soft chemical reduction. The details on growth process will be discussed.

Despite decades of research on cuprate superconductors, an understanding of what exact features of a material essentially support superconductivity has remained elusive. On an empirical basis, it seems natural to expect that superconductivity can be achieved through synthesis of cuprate analogs that share common features including spin one-half, two-dimensionality, and strong antiferromagnetic correlations etc [1]. Along this direction, Chaloupka et al. proposed that $\mathrm{LaNiO}_{3} / \mathrm{LaMO}_{3}$ (M stands for a trivalent cation Al, Ga, etc) superlattices would create superconductivity, which however has not been realized to date although the nickel-oxide superlattices have been successfully synthesized [2]. The recent report of superconductivity in an infinite-layer nickelate $\mathrm{Nd}_{0.8} \mathrm{Sr}_{0.2} \mathrm{NiO}_{2}$ has opened an alternative route to achieve superconductivity in transition metal oxide materials in addition to cuprates [3]. Compared with cuprates, the superconducting nickelates comprise a similar two-dimensional (2D) square lattice, isoelectronic $3 d^{9}$ valence state, and a superconducting dome in phase diagram [4 9]. But in sharp contrast to cuprates, the parent compound $\mathrm{NdNiO}_{2}$ of superconducting nickelates does not exhibit any magnetic order down to $1.7 \mathrm{~K}$ [10], indicating the underlying superconducting mechanism differs from that of cuprate superconductors for which magnetism are generally believed to be crucial for mediating Cooper pairs. Although various theoretical models have been proposed to explain the superconductivity in the superconducting nickelates [11 50], a consensus model has not yet reached partly owing to the challenges with the synthesis, and only very few reports exist on achieving superconducting films of infinite-layer nickelates [3, 5, 51/56]. At present, superconductivity has not been observed in bulk $\mathrm{Nd}_{1-x} \mathrm{Sr}_{x} \mathrm{NiO}_{2}[57-60]$.

In this paper, we report the successful growth of superconducting infinite-layer $\mathrm{Nd}_{0.8} \mathrm{Sr}_{0.2} \mathrm{NiO}_{2}$ films. The perovskite precursor phase nickelate films were prepared by using pulsed laser deposition (PLD). The infinite-layer phase was acquired by soft-chemistry 
reduction method. The thickness and out-plane x-ray diffraction (XRD) pattern of the prepared films were examined using a SmartLab x-ray diffractometer. The superconductivity was confirmed by transport measurements using a Quantum Design Physical Property Measurement System (PPMS) with a standard four-probe configuration. The wire connection was made by melting indium.

The laser target contained a stoichiometric mixture of $\mathrm{SrCO}_{3}$ (Alfa Aesar, 99.99\%), $\mathrm{Nd}_{2} \mathrm{O}_{3}$ (Alfa Aesar, 99.99\%), and NiO (Sigma Aldrich-Chemie GmbH, 99.995\%) prepared by a solid-state reaction in the air at $1100{ }^{\circ} \mathrm{C}$ for 24 hours. The products of this reaction were ground and reheated, and this process was repeated five times. The resulting polycrystalline materials was pressed into a pellet and sintered for 24 hours at $1200{ }^{\circ} \mathrm{C}$ in the air. The heating and cooling rate of sintering were kept at $3{ }^{\circ} \mathrm{C} / \mathrm{min}$.

The nickelate films were grown by PLD using 248-nm KrF excimer laser (COMPex 201, Coherent). The $\mathrm{SrTiO}_{3}$ (001) substrates $(5 * 5 \mathrm{~mm}$, without chemical etching to achieve a $\mathrm{TiO}_{2}$ terminated surface) were pre-annealed at $900{ }^{\circ} \mathrm{C}$ with an oxygen partial pressure of $1 \times 10^{-5}$ Torr. During growth, the substrate temperature was kept at $600{ }^{\circ} \mathrm{C}$ under an oxygen partial pressure of 150 mTorr. After deposition, the films were cooled to room temperature at a rate of $5{ }^{\circ} \mathrm{C}$ per minute in the same oxygen partial pressure. The laser beam size was about $0.8 \times 3.2 \mathrm{~mm}^{2}$ realized by using an aperture. The pulse energy of the laser was set to $430 \mathrm{~mJ}$ and $730 \mathrm{~mJ}$ for the growth of $\mathrm{NdNiO}_{3}$ and $\mathrm{Nd}_{0.8} \mathrm{Sr}_{0.2} \mathrm{NiO}_{3}$, respectively. The laser frequency was set to $4 \mathrm{~Hz}$.

The infinite-layer nickelate phase was acquired by soft-chemistry reduction method. As shown in Fig. 1 (b), the as-grown nickelate films were wrapped in clean aluminum foil and then sealed with $0.1 \mathrm{~g} \mathrm{CaH}_{2}$ powder (Alfa Aesar, 98\%) in quartz tubes which were pumped to a vacuum better than $1 \times 10^{-5}$ Torr. The reduction was carried out at a temperature of $290{ }^{\circ} \mathrm{C}$ for 5 hours, with the heating and cooling rates of $10{ }^{\circ} \mathrm{C} / \mathrm{min}$.

Figure 2 shows the structural characterization of the nickelate films. The $\mathrm{NdNiO}_{3}$ film peaks at $23.5^{\circ}$ and $48.2^{\circ}$ correspond to the (001) and (002) reflections, respectively (see Fig.2 (a)). After chemical reduction, as shown in Fig. 2 (b) the film peaks at $26.6^{\circ}$ and $55.7^{\circ}$ identify the infinite-layer $\mathrm{NdNiO}_{2}$ and correspond to (001) and (002) reflections, respectively. For a typical film of $\mathrm{Nd}_{0.8} \mathrm{Sr}_{0.2} \mathrm{NiO}_{3}$ shown in Fig. 2(c), the peaks at $23.8^{\circ}$ and $48.4^{\circ}$ correspond to (001) and (002) reflections, respectively, and both two peaks are slightly broader than those of undoped phase in Fig. 2 (a). After chemical reduction, the peaks at 
$26.5^{\circ}$ and $54.8^{\circ}$ correspond to (001) and (002) reflections, respectively, as expected for a film of an infinite-layer $\mathrm{Nd}_{0.8} \mathrm{Sr}_{0.2} \mathrm{NiO}_{2}$ (Fig.2(d)). The intensity is comparable to the precursor. It was reported that the $\mathrm{Nd}_{0.8} \mathrm{Sr}_{0.2} \mathrm{NiO}_{3}$ films were difficult to grow due to the formation of a secondary phase which shows only a single peak with $2 \theta$ less than $48^{\circ}$ [52].

In Fig 3 we show the measured $\mathrm{Nd}_{0.8} \mathrm{Sr}_{0.2} \mathrm{NiO}_{2}$ film resistance as a function of temperature revealing that a superconducting transition occurs below $9 \mathrm{~K}$ and the zero resistivity is achieved at about $3.5 \mathrm{~K}$. The superconducting transition temperature of our film is a little lower than that reported in the literature, which probably stems from the variation of the hole concentration in the films prepared under differing growth and reduction conditions. To study the stability of the superconducting $\mathrm{Nd}_{0.8} \mathrm{Sr}_{0.2} \mathrm{NiO}_{2}$ films, we measured the resistivity of a typical film which had been kept in the glovebox for 30 days after chemical reduction and had been exposed to air longer than 10 hours during XRD and other measurements. The superconducting transition is almost identical with the same critical temperature $T_{c}$, as shown in Fig. 3(c). It seems that the 8.5nm-thick superconducting film without capping layer is fairly stable, promising the suitability for multiple experiments that require stable samples.

Currently, using the PLD growth and soft chemical reduction procedure introduced above, we manage to produce at least one superconducting sample out of the ten films, and we are continuing our optimization of the growth condition to improve even higher the reproducibility. The growth window is extremely narrow probably because the formation energy of the desired 113 phase differs only slightly from that of the secondary phase. Nevertheless, we find that the XRD pattern of the precursor 113 phase is a good criterion to obtain superconducting films. The (002) peak of the secondary phase locates at about $47.8^{\circ}$, while for 113 phase the (002) peak is at the $2 \theta$ larger than $48^{\circ}$ and thickness dependent. In the optimization, the $2 \theta$ of the (002) reflection varies almost continuously from $47.8^{\circ}$ to $48.5^{\circ}$. For a typical 8.5nm-thick $\mathrm{Nd}_{0.8} \mathrm{Sr}_{0.2} \mathrm{NiO}_{3}$ film, we find that as long as the (002) peak reaches $48.5^{\circ}$, the superconducting phase is easily achieved through chemical reduction; and if the (002) peak locates at $48.3^{\circ}$, the superconductivity can be achieved, though it is much harder to be turned into the infinite-layer phase using the chemical reduction, and the corresponding critical temperature Tc varies from $5 \mathrm{~K}$ to $9 \mathrm{~K}$. However, the precursor films with $2 \theta$ of the (002) peak less than $48^{\circ}$ have never been reduced to the infinite-layer phase, let alone the superconducting phase. 
In summary, we have successfully synthesized superconducting infinite-layer $\mathrm{Nd}_{0.8} \mathrm{Sr}_{0.2} \mathrm{NiO}_{2}$ thin films by using pulsed laser deposition and soft-chemistry reduction method. The details on the film growth and subsequent chemical reduction process are discussed. Our results provide important information for the preparation of superconducting nickelate films that is a crucial step in this field.

\section{Acknowledgement}

We wish to thank Er-jia Guo for helpful discussions. This work was supported in part by the National Natural Science Foundation of China (Grant No. 12074411) and (Grant No. 11888101), the National Key Research and Development Program of China (Grant No. 2016YFA0300300 and 2017YFA0302900), the Strategic Priority Research Program (B) of the Chinese Academy of Sciences (Grant No. XDB25000000) and the Research Program of Beijing Academy of Quantum Information Sciences (Grant No. Y18G06).

\section{Author Contributions}

Z.H.Z., X.J.Z., and Q.G. proposed and designed the research. Q.G., Y.C.Z. and Z.H.Z. grew the films. Q.G. carried out the XRD and transport measurements with the help from Y.C.Z. Z.H.Z. and Q.G. wrote the paper. All authors participated in discussions and comments on the paper.

[1] Jiří Chaloupka and Giniyat Khaliullin. Orbital order and possible superconductivity in $\mathrm{LaNiO}_{3} / \mathrm{LaMO}_{3}$ superlattices. Physical Review Letters, 100(1):016404, 2008.

[2] A. V. Boris, Y. Matiks, E. Benckiser, A. Frano, P. Popovich, V. Hinkov, P. Wochner, M. Castro-Colin, E. Detemple, V. K. Malik, C. Bernhard, T. Prokscha, A. Suter, Z. Salman, E. Morenzoni, G. Cristiani, H. U. Habermeier, and B. Keimer. Dimensionality Control of Electronic Phase Transitions in Nickel-Oxide Superlattices. Science, 332(6032):937-940, 2011.

[3] D. F. Li, K. Lee, B. Y. Wang, M. Osada, S. Crossley, H. R. Lee, Y. Cui, Y. Hikita, and H. Y. Hwang. Superconductivity in an infinite-layer nickelate. Nature, 572(7771):624-+, 2019.

[4] D. F. Li, B. Y. Wang, K. Lee, S. P. Harvey, M. Osada, B. H. Goodge, L. F. Kourkoutis, and H. Y. Hwang. Superconducting Dome in $\mathrm{Nd}_{1-x} \mathrm{Sr}_{x} \mathrm{NiO}_{2}$ Infinite Layer Films. Physical Review Letters, 125(2):6, 2020. 
[5] S. W. Zeng, C. S. Tang, X. M. Yin, C. J. Li, M. S. Li, Z. Huang, J. X. Hu, W. Liu, G. J. Omar, H. Jani, Z. S. Lim, K. Han, D. Y. Wan, P. Yang, S. J. Pennycook, A. T. S. Wee, and A. Ariando. Phase Diagram and Superconducting Dome of Infinite-Layer $\mathrm{Nd}_{1-x} \mathrm{Sr}_{x} \mathrm{NiO}_{2} \mathrm{Thin}$ Films. Physical Review Letters, 125(14):7, 2020.

[6] M. Osada, B. Y. Wang, K. Lee, D. F. Li, and H. Y. Hwang. Phase diagram of infinite layer praseodymium nickelate $\mathrm{Pr}_{1-x} \mathrm{Sr}_{x} \mathrm{NiO}_{2}$ thin films. Physical Review Materials, 4(12):5, 2020.

[7] M. Hepting, D. Li, C. J. Jia, H. Lu, E. Paris, Y. Tseng, X. Feng, M. Osada, E. Been, Y. Hikita, Y. D. Chuang, Z. Hussain, K. J. Zhou, A. Nag, M. Garcia-Fernandez, M. Rossi, H. Y. Huang, D. J. Huang, Z. X. Shen, T. Schmitt, H. Y. Hwang, B. Moritz, J. Zaanen, T. P. Devereaux, and W. S. Lee. Electronic structure of the parent compound of superconducting infinite-layer nickelates. Nature Materials, 19(4):381-+, 2020.

[8] B. H. Goodge, D. F. Li, K. Lee, M. Osada, B. Y. Wang, G. A. Sawatzky, H. Y. Hwang, and L. F. Kourkoutis. Doping evolution of the Mott-Hubbard landscape in infinite-layer nickelates. Proceedings of the National Academy of Sciences of the United States of America, 118(2):7, 2021.

[9] Matteo Rossi, Haiyu Lu, Abhishek Nag, Danfeng Li, Motoki Osada, Kyuho Lee, Bai Yang Wang, Stefano Agrestini, M Garcia-Fernandez, and Y-D Chuang. Orbital and Spin Character of Doped Carriers in Infinite-Layer Nickelates. arXiv:2011.00595, 2020.

[10] MA Hayward and MJ Rosseinsky. Synthesis of the infinite layer $\mathrm{Ni}$ (I) phase $\mathrm{NdNiO}_{2+x}$ by low temperature reduction of $\mathrm{NdNiO}_{3}$ with sodium hydride. Solid state sciences, 5(6):839-850, 2003.

[11] A. S. Botana and M. R. Norman. Similarities and Differences between $\mathrm{LaNiO}_{2}$ and $\mathrm{CaCuO}_{2}$ and Implications for Superconductivity. Physical Review X, 10(1):6, 2020.

[12] J. E. Hirsch and F. Marsiglio. Hole superconductivity in infinite-layer nickelates. Physica C-Superconductivity and Its Applications, 566:3, 2019.

[13] P. H. Jiang, L. Si, Z. L. Liao, and Z. C. Zhong. Electronic structure of rare-earth infinite-layer $\mathrm{RNiO}_{2}(\mathrm{R}=\mathrm{La}, \mathrm{Nd})$. Physical Review B, 100(20):5, 2019.

[14] Y. Nomura, M. Hirayama, T. Tadano, Y. Yoshimoto, K. Nakamura, and R. Arita. Formation of a two-dimensional single-component correlated electron system and band engineering in the nickelate superconductor $\mathrm{NdNiO}_{2}$. Physical Review B, 100(20):11, 2019.

[15] G. M. Zhang, Y. F. Yang, and F. C. Zhang. Self-doped Mott insulator for parent compounds 
of nickelate superconductors. Physical Review B, 101(2):5, 2020.

[16] P. Adhikary, S. Bandyopadhyay, T. Das, I. Dasgupta, and T. Saha-Dasgupta. Orbitalselective superconductivity in a two-band model of infinite-layer nickelates. Physical Review $B, 102(10): 5,2020$.

[17] J. Karp, A. S. Botana, M. R. Norman, H. Park, M. Zingl, and A. Millis. Many-Body Electronic Structure of $\mathrm{NdNiO}_{2}$ and $\mathrm{CaCuO}_{2}$. Physical Review X, 10(2):11, 2020.

[18] S. Bandyopadhyay, P. Adhikary, T. Das, I. Dasgupta, and T. Saha-Dasgupta. Superconductivity in infinite-layer nickelates: Role of f orbitals. Physical Review B, 102(22):6, 2020.

[19] J. Chang, J. Z. Zhao, and Y. Ding. Hund-Heisenberg model in superconducting infinite-layer nickelates. European Physical Journal B, 93(12):7, 2020.

[20] M. Y. Choi, K. W. Lee, and W. E. Pickett. Role of 4 f states in infinite-layer $\mathrm{NdNiO}_{2}$. Physical Review B, 101(2):7, 2020.

[21] B. Geisler and R. Pentcheva. Fundamental difference in the electronic reconstruction of infinite-layer versus perovskite neodymium nickelate films on $\mathrm{SrTiO}_{2}(001)$. Physical Review $B, 102(2): 6,2020$.

[22] Yuhao Gu, Sichen Zhu, Jiangping Hu, and Hanghui Chen. Hybridization and correlation effects in the electronic structure of infinite-layer nickelates. Bulletin of the American Physical Society, 65, 2020.

[23] Y. H. Gu, S. C. Zhu, X. X. Wang, J. P. Hu, and H. H. Chen. A substantial hybridization between correlated Ni-d orbital and itinerant electrons in infinite-layer nickelates. Communications Physics, 3(1):9, 2020.

[24] R. He, P. H. Jiang, Y. Lu, Y. D. Song, M. X. Chen, M. L. Jin, L. L. Shui, and Z. C. Zhong. Polarity-induced electronic and atomic reconstruction at $\mathrm{NdNiO}_{2} / \mathrm{SrTiO}_{2}$ interfaces. Physical Review B, 102(3):7, 2020.

[25] C. Ji, G. Wu, S. W. Yang, and X. P. Yang. Completely polarized e(g) orbitals realized in d(7)-Ni ${ }^{3+}$ based heterointerface. Physical Review Materials, 4(12):7, 2020.

[26] M. Jiang, M. Berciu, and G. A. Sawatzky. Critical Nature of the Ni Spin State in Doped $\mathrm{NdNiO}_{2}$. Physical Review Letters, 124(20):5, 2020.

[27] J. Kapeghian and A. S. Botana. Electronic structure and magnetism in infinite-layer nickelates $\mathrm{RNiO}_{2}(\mathrm{R}=\mathrm{La}-\mathrm{Lu})$. Physical Review B, 102(20):14, 2020.

[28] V. M. Katukuri, N. A. Bogdanov, O. Weser, J. van den Brink, and A. Alavi. Electronic 
correlations and magnetic interactions in infinite-layer $\mathrm{NdNiO}_{2}$. Physical Review B, 102(24):7, 2020 .

[29] J. Krishna, H. LaBollita, A. O. Fumega, V. Pardo, and A. S. Botana. Effects of Sr doping on the electronic and spin-state properties of infinite-layer nickelates: Nature of holes. Physical Review B, 102(22):7, 2020.

[30] Zi-Jian Lang, Ruoshi Jiang, and Wei Ku. Where do the doped hole carriers reside in the new superconducting nickelates? arXiv:2005.00022, 2020.

[31] Jiacheng Gao, Zhijun Wang, Chen Fang, and Hongming Weng. Electronic structures and topological properties in nickelates $L n_{n+1} \mathrm{Ni}_{n} \mathrm{O}_{2 n+2}$. arXiv:1909.04657, 2019.

[32] F. Lechermann. Multiorbital Processes Rule the $\mathrm{Nd}_{1-x} \mathrm{Sr}_{x} \mathrm{NiO}_{2}$ Normal State. Physical Review $X, 10(4): 14,2020$.

[33] F. Lechermann. Late transition metal oxides with infinite-layer structure: Nickelates versus cuprates. Physical Review B, 101(8):5, 2020.

[34] I. Leonov, S. L. Skornyakov, and S. Y. Savrasov. Lifshitz transition and frustration of magnetic moments in infinite-layer $\mathrm{NdNiO}_{2}$ upon hole doping. Physical Review B, 101(24):5, 2020.

[35] Z. Liu, Z. Ren, W. Zhu, Z. F. Wang, and J. L. Yang. Electronic and magnetic structure of infinite-layer $\mathrm{NdNiO}_{2}$ : trace of antiferromagnetic metal. Npj Quantum Materials, 5(1):8, 2020 .

[36] E. M. Nica, J. Krishna, R. Yu, Q. M. Si, A. S. Botana, and O. Erten. Theoretical investigation of superconductivity in trilayer square-planar nickelates. Physical Review B, 102(2):6, 2020.

[37] F. Petocchi, V. Christiansson, F. Nilsson, F. Aryasetiawan, and P. Werner. Normal State of $\mathrm{Nd}_{1-x} \mathrm{Sr}_{x} \mathrm{NiO}_{2}$ from Self-Consistent GW plus EDMFT. Physical Review X, 10(4):9, 2020.

[38] S. Ryee, H. Yoon, T. J. Kim, M. Y. Jeong, and M. J. Han. Induced magnetic twodimensionality by hole doping in the superconducting infinite-layer nickelate $\mathrm{Nd}_{1-x} \mathrm{Sr}_{x} \mathrm{NiO}_{2}$. Physical Review B, 101(6):5, 2020.

[39] H. Sakakibara, H. Usui, K. Suzuki, T. Kotani, H. Aoki, and K. Kuroki. Model Construction and a Possibility of Cupratelike Pairing in a New d(9) Nickelate Superconductor (Nd,Sr) $\mathrm{NiO}_{2}$. Physical Review Letters, 125(7):6, 2020.

[40] L. Si, W. Xiao, J. Kaufmann, J. M. Tomczak, Y. Lu, Z. C. Zhong, and K. Held. Topotactic Hydrogen in Nickelate Superconductors and Akin Infinite-Layer Oxides ABO(2). Physical Review Letters, 124(16):8, 2020. 
[41] Y. Wang, C. J. Kang, H. Miao, and G. Kotliar. Hund's metal physics: From $\mathrm{SrNiO}_{2}$ to $\mathrm{LaNiO}_{2}$. Physical Review B, 102(16):8, 2020.

[42] Z. Wang, G. M. Zhang, Y. F. Yang, and F. C. Zhang. Distinct pairing symmetries of superconductivity in infinite-layer nickelates. Physical Review B, 102(22):6, 2020.

[43] P. Werner and S. Hoshino. Nickelate superconductors: Multiorbital nature and spin freezing. Physical Review B, 101(4):5, 2020.

[44] X. X. Wu, D. Di Sante, T. Schwemmer, W. Hanke, H. Y. Hwang, S. Raghu, and R. Thomale. Robust $\mathrm{dx}^{2}-\mathrm{y}^{2}$-wave superconductivity of infinite-layer nickelates. Physical Review B, 101(6):5, 2020.

[45] Xianxin Wu, Kun Jiang, Domenico Di Sante, Werner Hanke, Jiangping Hu, and Ronny Thomale. Surface $s$-wave superconductivity for oxide-terminated infinite-layer nickelates. arXiv:2008.06009, 2020.

[46] Ruiqi Zhang, Christopher Lane, Bahadur Singh, Johannes Nokelainen, Bernardo Barbiellini, Robert S Markiewicz, Arun Bansil, and Jianwei Sun. f-electron and magnetic ordering effects in nickelates $\mathrm{LaNiO}_{2}$ and $\mathrm{NdNiO}_{2}$ : remarkable role of the cuprate-like $3 d_{x^{2}-y^{2}}$ band. arXiv:2009.05816, 2020.

[47] Y. Zhang, L. F. Lin, W. J. Hu, A. Moreo, S. Dong, and E. Dagotto. Similarities and differences between nickelate and cuprate films grown on a $\mathrm{SrTiO}_{3}$ substrate. Physical Review B, 102(19):10, 2020.

[48] Michael Klett, Tilman Schwemmer, Sebastian Wolf, Xianxin Wu, David Riegler, Andreas Dittmaier, Domenico Di Sante, Gang Li, Werner Hanke, and Stephan Rachel. From high- $T_{c}$ to low- $T_{c}$ : Multi-orbital effects in transition metal oxides. arXiv:2101.04689, 2021.

[49] Z. Liu, C. C. Xu, C. Cao, W. Zhu, Z. F. Wang, and J. L. Yang. Doping dependence of electronic structure of infinite-layer $\mathrm{NdNiO}_{2}$. Physical Review B, 103(4):10, 2021.

[50] Xiangang Wan, Vsevolod Ivanov, Giacomo Resta, Ivan Leonov, and Sergey Y. Savrasov. Exchange interactions and sensitivity of the Ni two-hole spin state to Hund's coupling in doped $\mathrm{NdNiO}_{2}$. Physical Review B, 103(7):075123, 2021.

[51] M. Osada, B. Y. Wang, B. H. Goodge, K. Lee, H. Yoon, K. Sakuma, D. F. Li, M. Miura, L. F. Kourkoutis, and H. Y. Hwang. A Superconducting Praseodymium Nickelate with Infinite Layer Structure. Nano Letters, 20(8):5735-5740, 2020.

[52] K. Lee, B. H. Goodge, D. F. Li, M. Osada, B. Y. Wang, Y. Cui, L. F. Kourkoutis, and H. Y. 
Hwang. Aspects of the synthesis of thin film superconducting infinite-layer nickelates. Apl Materials, 8(4):11, 2020.

[53] Q. Q. Gu, Y. Y. Li, S. Y. Wan, H. Z. Li, W. Guo, H. Yang, Q. Li, X. Y. Zhu, X. Q. Pan, Y. F. Nie, and H. H. Wen. Single particle tunneling spectrum of superconducting $\mathrm{Nd}_{1-x} \mathrm{Sr}_{x} \mathrm{NiO}_{2}$ thin films. Nature Communications, 11(1):7, 2020.

[54] Ying Xiang, Qing Li, Yueying Li, Huan Yang, Yuefeng Nie, and Hai-Hu Wen. Magnetic transport properties of superconducting $\mathrm{Nd}_{1-x} \mathrm{Sr}_{x} \mathrm{NiO}_{2}$ thin films. arXiv:2007.04884, 2020.

[55] B. Y. Wang, D. F. Li, B. H. Goodge, K. Lee, M. Osada, S. P. Harvey, L. F. Kourkoutis, M. R. Beasley, and H. Y. Hwang. Isotropic Pauli-limited superconductivity in the infinite-layer nickelate $\mathrm{Nd}_{0.775} \mathrm{Sr}_{0.225} \mathrm{NiO}_{2}$. Nature Physics, page 6, 2021.

[56] X. R. Zhou, Z. X. Feng, P. X. Qin, H. Yan, S. Hu, H. X. Guo, X. N. Wang, H. J. Wu, X. Zhang, H. Y. Chen, X. P. Qiu, and Z. Q. Liu. Absence of superconductivity in Nd0.8Sr0.2NiOx thin films without chemical reduction. Rare Metals, 39(4):368-374, 2020.

[57] Qing Li, Chengping He, Jin Si, Xiyu Zhu, Yue Zhang, and Hai-Hu Wen. Absence of superconductivity in bulk $\mathrm{Nd}_{1-x} \mathrm{Sr}_{x} \mathrm{NiO}_{2}$. Communications Materials, 1(1), 2020.

[58] B. X. Wang, H. Zheng, E. Krivyakina, O. Chmaissem, P. P. Lopes, J. W. Lynn, L. C. Gallington, Y. Ren, S. Rosenkranz, J. F. Mitchell, and D. Phelan. Synthesis and characterization of bulk $\mathrm{Nd}_{1-x} \mathrm{~S}_{x} \mathrm{NiO}_{2}$ and $\mathrm{Nd}_{1-x} \mathrm{~S}_{x} \mathrm{NiO}_{3}$. Physical Review Materials, 4(8):9, 2020.

[59] Chengping He, Xue Ming, Qing Li, Xiyu Zhu, Jin Si, and Hai-Hu Wen. Synthesis and physical properties of perovskite $\mathrm{Sm}_{1-x} \mathrm{Sr}_{x} \mathrm{NiO}_{3}(\mathrm{x}=0,0.2)$ and infinite-layer $\mathrm{Sm}_{0.8} \mathrm{Sr}_{0.2} \mathrm{NiO}_{2}$ nickelates. arXiv:2010.11777, 2020.

[60] Yi Cui, C Li, Q Li, Xiyu Zhu, Z Hu, Yi-feng Yang, JS Zhang, Rong Yu, Hai-Hu Wen, and Weiqiang Yu. NMR Evidence of Antiferromagnetism in $\mathrm{Nd}_{0.85} \mathrm{Sr}_{0.15} \mathrm{NiO}_{2}$. arXiv:2011.09610, 2020 . 
(a)

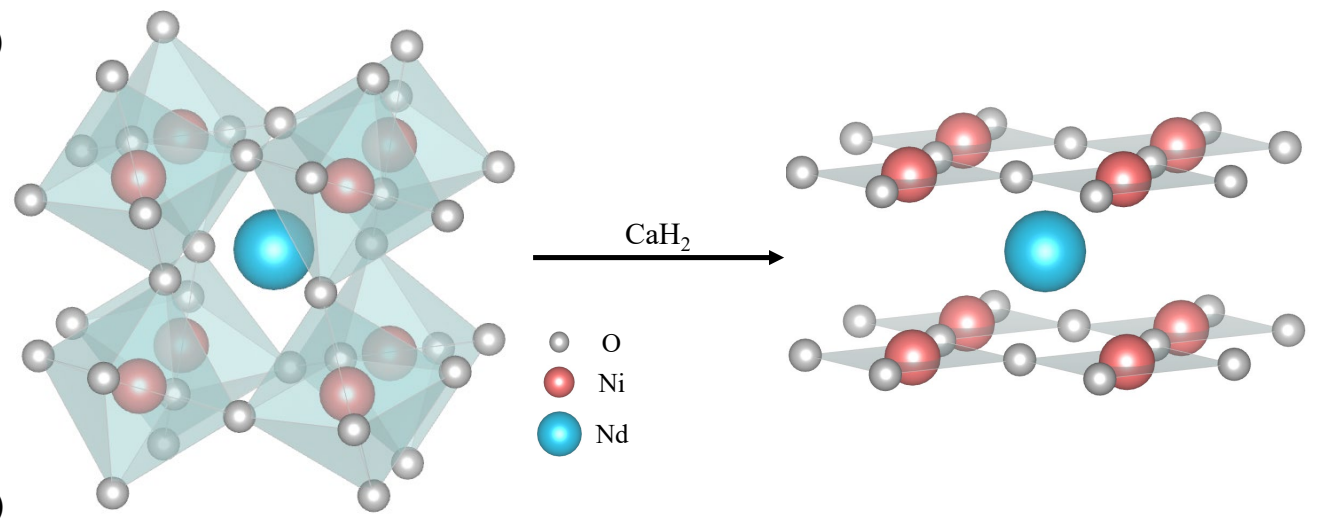

(b)

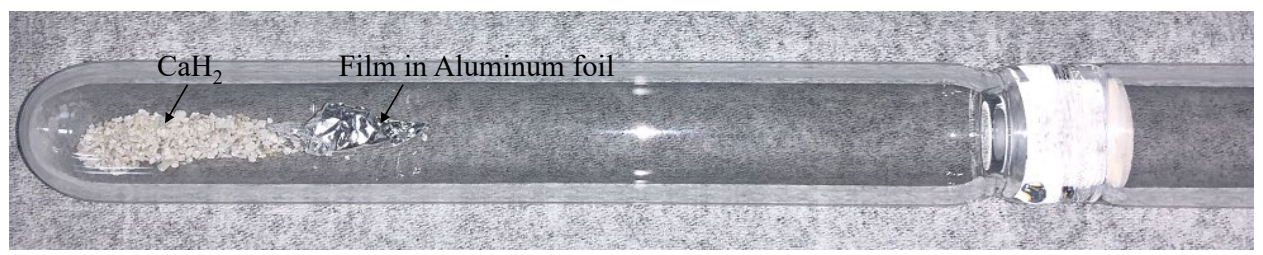

FIG. 1: Crystal structure and the reduction process of nickelates. (a) illustrates the crystal structures of $\mathrm{NdNiO}_{3}$ (left) and $\mathrm{NdNiO}_{2}$ (right). (b) The soft-chemistry reduction process. A typical $\mathrm{Nd}_{0.8} \mathrm{Sr}_{0.2} \mathrm{NiO}_{3}$ film wrapped in Aluminum foil is vacuum-sealed with $\mathrm{CaH}_{2}$ powder in a quartz tube. 

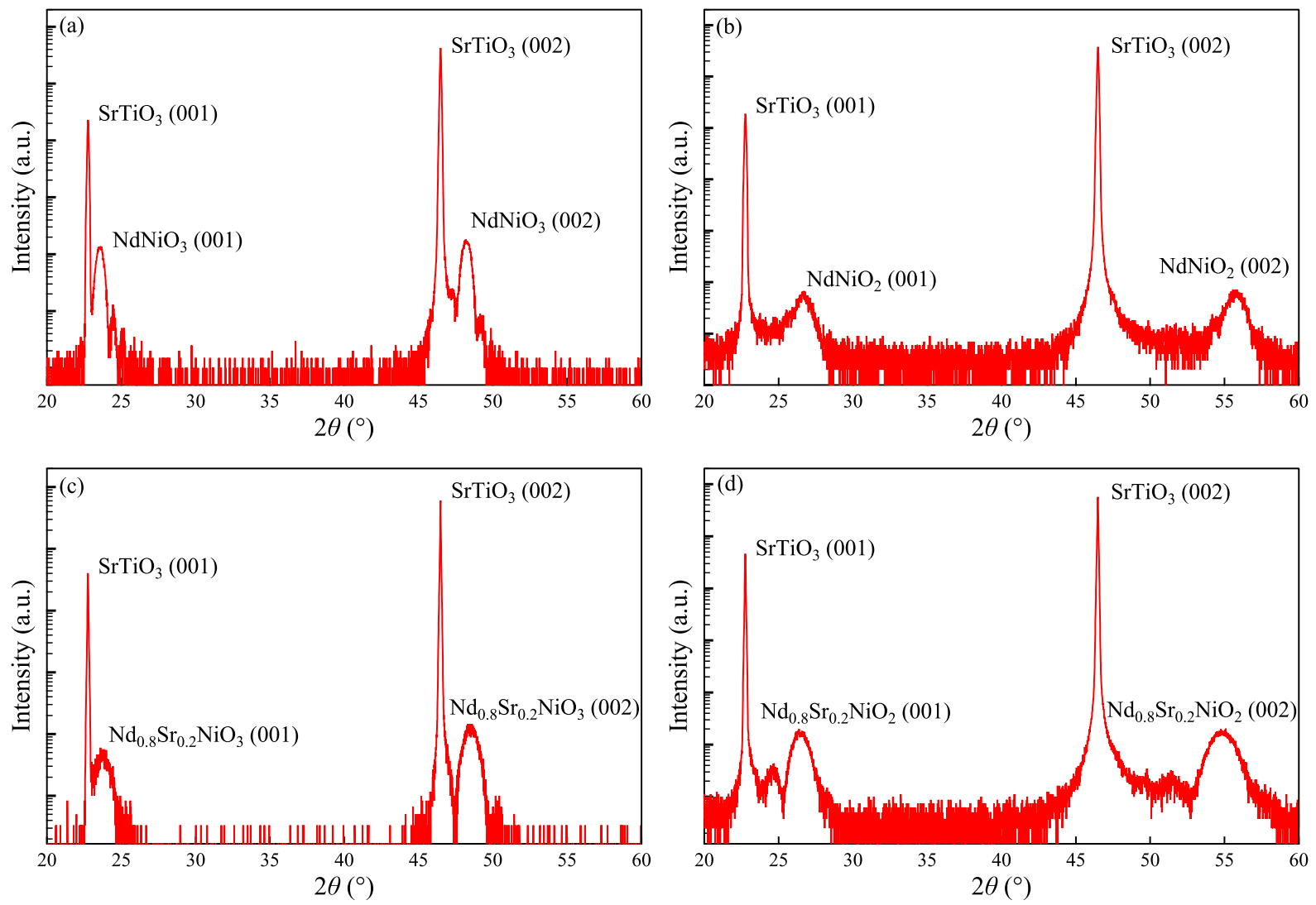

FIG. 2: Structural characterization of nickelate films on STO substrates. (a) The XRD $\theta-2 \theta$ scans of a typical $\mathrm{NdNiO}_{3}$ film with a thickness of $14 \mathrm{~nm}$. (b) The XRD $\theta-2 \theta$ scans of the infinite-layer $\mathrm{NdNiO}_{2}$ film acquired by performing chemical reduction on the sample (a) at 290 ${ }^{\circ} \mathrm{C}$ for 5 hours. (c) The XRD $\theta-2 \theta$ scans of a typical $\mathrm{Nd}_{0.8} \mathrm{Sr}_{0.2} \mathrm{NiO}_{3}$ film with a thickness of 9 nm. (d) The XRD $\theta-2 \theta$ scans of the infinite-layer $\mathrm{Nd}_{0.8} \mathrm{Sr}_{0.2} \mathrm{NiO}_{2}$ film acquired by reducing the sample (c) at $290{ }^{\circ} \mathrm{C}$ for 5 hours. 

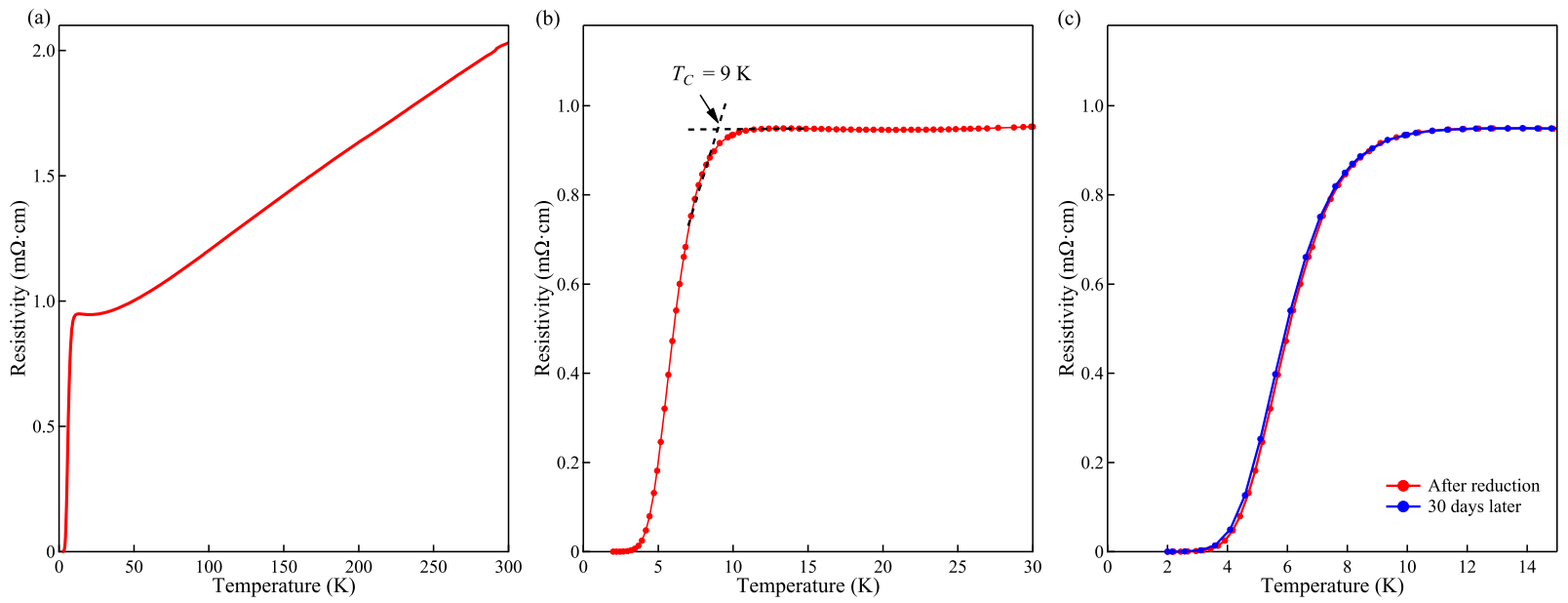

FIG. 3: Transport properties of $\mathrm{Nd}_{0.8} \mathbf{S r}_{0.2} \mathbf{N i O}_{2}$ thin film. (a) Temperature-dependent resistivity of the $\mathrm{Nd}_{0.8} \mathrm{Sr}_{0.2} \mathrm{NiO}_{2}$ thin film from $300 \mathrm{~K}$ to $2 \mathrm{~K}$. (b) A zoom-in view of (a) with the temperature lower than $30 \mathrm{~K}$. The onset of the superconducting transition is about $9 \mathrm{~K}$. (c) An aging test of a nickelate superconducting film. The transport measurement shows almost identical superconducting transition, over a period of 30 days after the chemical reduction. 